\title{
$\left(\mathrm{Bi}_{1.6} \mathrm{~Pb}_{0.4}\right) \mathrm{Sr}_{2} \mathrm{Ca}_{2} \mathrm{Cu}_{3} \mathrm{O}_{y}$ 中 ${ }^{63} \mathrm{Cu}$ 核磁共 振的变温研究 ${ }^{*}$
}

许亮李建奇 边明华 邬学文

(华东师范大学分析测试中心, 上海 200062)

\section{关篗词 $\left(\mathrm{Bi}_{1.6} \mathrm{~Pb}_{0.4}\right) \mathrm{Sr}_{2} \mathrm{Ca}_{2} \mathrm{Cu}_{3} \mathrm{O}_{y}$ 磁取向样品、 ${ }^{63} \mathrm{Cu}$ 核磁共振、奈特位移、核自旋一晶格驰理率}

从 1987 年以来, 人们对高临界温度超导材料作了大量的核磁共振 (NMR) 测量, 主要集中在 Y系 123 相的单晶和磁取向样品上. 发现这类超导体不能用传统的 Fermi 液体物理图象来理 解其在正常态的 NMR 行为, 以及在超导态时表现出非常规电声配对机制. 由此引出的分析和 讨论都对各种理论模型作了实验上的限制 ${ }^{[1]}$. 由于 $\mathrm{Bi}$ 系氧化物超导体存在调制结构, 在化学 均匀性和单相性方面都比 $Y$ 系差. 这样每个晶格位置的化学环境有很大的分布, 会引起 NMR 谱线的增宽. 这种材料本征结构上的特点限制了它的 NMR 研究, 特别是 Bi 系 2223 相, 因为单相样品难于得到, 有关这种材料中铜的 NMR 研究至今报道甚少.

我们制备了掺铅 $\mathrm{Bi}$ 系 2223 相单相 (X 射线衍射谱误差范围内) 磁取向样品. 在 77一 $295 \mathrm{~K}$ 范围内, 晶粒 $c$ 轴平行和垂直于磁场的两种情况下, 测量了 $\mathrm{Cu}(1)$ 位, $\mathrm{Cu}(2)$ 位的 核自旋 - 晶格弛豫率和奈特 (Knight) 位移的变温规律, 并对这些实验结果作详细的讨论.

\section{1 样品制备和实验方法}

用常规的固相反应法制备掺铅 $\mathrm{Bi}$ 系 2223 相块状粉末样品: 以分析纯 $\mathrm{Bi}_{2} \mathrm{O}_{3}, \mathrm{SrCO}_{3}, \mathrm{CaCO}_{2}$, $\mathrm{PbO}$ 和 $\mathrm{CuO}$ 为原料, 按标称成份 $\left(\mathrm{Bi}_{1.6} \mathrm{~Pb}_{0.4}\right) \mathrm{Sr}_{2} \mathrm{Ca}_{2} \mathrm{Cu}_{3} \mathrm{O}_{y}$ 配料, 原料经球磨混匀, 在 $800{ }^{\circ} \mathrm{C}$ 空气 中预烧 2 次, 各 $24 \mathrm{~h}$. 再经过压饼 $\left(850 \mathrm{~kg} / \mathrm{cm}^{2}\right)$, 在 $852^{\circ} \mathrm{C}$ 空气中烧结 2 次, 各 $150 \mathrm{~h} . X$ 射线衍 射谱 (XRD) 均未发现杂相 (2212 相, 2201 相和 $\left.\mathrm{Ca}_{2} \mathrm{PbO}_{5}\right)$ 和杂质的特征峰. 用交流磁化率测得 零磁场临界转变温度 $T_{\mathrm{c}}=108 \mathrm{~K}$.

块状样品用无水酒精研磨 $0.25-0.5 \mathrm{~h}$, 通过 600 目网管, 晶粒尺寸小于 $25 \mu \mathrm{m}$. XRD 谱未 发现非晶化增宽. 样品在 $B_{0}=T \mathrm{~T}$ 的磁场下磁取向固化, 方法见文献[2]. 晶粒 $\mathrm{c}$ 轴垂直面的 XRD 谱中非 $(001)$ 峰几乎都被抑制, 说明晶粒 $c$ 轴沿磁场取向排列. 利用 NMR 探头线圈 $Q$ 值的变化 ${ }^{[3]}$, 在 $B_{o}=T \mathrm{~T}$ 的磁场下测得 $T_{\mathrm{c}}=(100 \pm 1) \mathrm{K}$.

NMR 实验都在 Bruker 公司生产的 MSL-300 型富氏变换核磁共振谱仪上进行. 磁场为 $B_{0}=7 \mathrm{~T}$. 变温实验用自制的液氮变温 NMR 探头 ${ }^{[4]}$, 温度变化范围为 $77-295 \mathrm{~K}$, 温控仪为 Bruker 公司的 B-VT1000 型温控器. 以样品中 ${ }^{63} \mathrm{Cu}$ 为测量对象, 在测量不同晶位和取向时, 射 频范围在 $79.8-81.5 \mathrm{MHz}$ 之间.

1993-11-30 收稿, 1994-06-10 收修改稿.

*上海市自然科学基金及国家教委博士点基金和国家超导中心资助课题. 


\section{2 实验结果和数据处理}

\section{1 觧态 ${ }^{6} \mathrm{Cu}$ NMR 橎}

图 1 是 $140 \mathrm{~K}$ 温度下用自旋回波逐点扫频方法得到的 $c$ 轴 $/ / B_{0}, c$ 轴 $\perp B_{0}$ 二种情况下的 $\mathrm{Cu}(1), \mathrm{Cu}(2)$ 位的静态谱. 自旋回波峰值对应于样品的磁化强度矢量, 取回波峰值作纵坐 标. 其中 $c$ 轴 $/ / B_{0}$ 时, $\mathrm{Cu}(1)$ 和 $\mathrm{Cu}(2)$ 位的谱线在整个温度变化范围内是重叠的. 这些谱线半 高宽为 $150-300 \mathrm{kHz}$, 取谱线高度 $90 \%$ 处的宽度 $(20-40 \mathrm{kHz})$ 为误差范围, 折算成 Knight 位 移的表示, 在 $0.025 \%-0.050 \%$ 范围内.

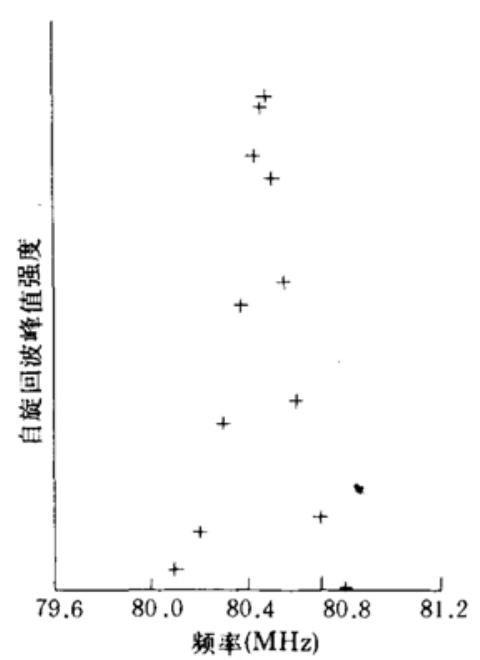

(a)

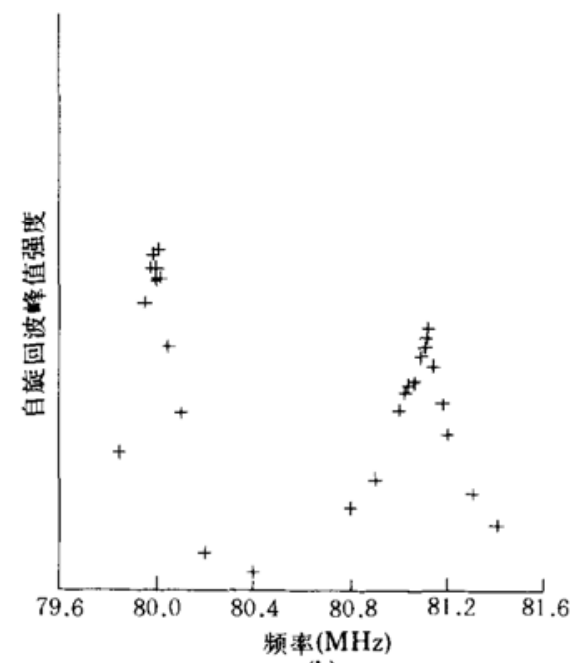

(b)

图 $1\left(\mathrm{Bi}_{1.6} \mathrm{~Pb}_{0.4}\right) \mathrm{Sr}_{2} \mathrm{Ca}_{2} \mathrm{Cu}_{3} \mathrm{O}_{y}$ 磁取向样品的 ${ }^{63} \mathrm{Cu}$ NMR 谱

(a) 晶粒 $c$ 轴平行于磁场时, $\mathrm{Cu}(1)$ 位和 $\mathrm{Cu}(2)$ 位谱线重春; (b) 晶粒 $c$ 轴垂直于磷场时, $80.1 \mathrm{MHz}$ 处为 $\mathrm{Cu}(1)$ 位橧线, $81.1 \mathrm{MHz}$ 处为 $\mathrm{Cu}(2)$ 位橧 线

\subsection{Knight 位移各向异性数的确定}

在磁场下四极核 (例如 $I=3 / 2$ 的 ${ }^{63} \mathrm{Cu}$ ) 的核自旋哈密顿量包含电子超精细相互作用和电四 极矩相互作用, 磁取向样品相对于磁场的 2 种不同取向时, 二阶微扰论可给出谱线的频率位置 表示 ${ }^{[2]}$ :

$$
\begin{aligned}
& c \text { 轴// } B_{0} \text { 时, } v_{/ /}=v_{0}\left(1+K_{/ /}\right)+v_{Q}^{2} \eta /\left(12 v_{0}\right), \\
& c \text { 轴 } \perp B_{0} \text { 时, } v_{\perp}=v_{0}\left(1+K_{\perp}\right)+v_{Q}^{2}(3-\eta)^{2} /\left(48 v_{0}\right) .
\end{aligned}
$$

由于样品晶体结构的四方对称性, $a, b$ 轴是不可区分的, 即 $K_{a a}=K_{b b}=K_{\perp}, K$ 是 Knight 位 移. 在 $c$ 轴 $/ / B_{0}$ 时, 发现 ${ }^{65} \mathrm{Cu}$ 和 ${ }^{63} \mathrm{Cu}$ 谱线的频率之比等于它们的旋磁比 $(\gamma)$ 之比值, 即 ${ }^{65} v_{/ /} /{ }^{63} v_{/ /}=$ ${ }^{65} \gamma /{ }^{63} \gamma=1.07$. 由于裸核或绝缘体的 NMR 共振频率 $v_{0}$ 与旋磁比 $\gamma$ 成正比, 结合晶体结构中 $\mathrm{Cu}(1)$ 和 $\mathrm{Cu}(2)$ 位是关于 $c$ 轴对称的, 并假设 $\mathrm{Knight}$ 位移的同位素效应很小 ${ }^{(9)}$, 由此可认 为, $\mathrm{Cu}(1)$ 位和 $\mathrm{Cu}(2)$ 位的电场梯度 (EFG) 张量的不对称参数 $\eta \approx 0$. (1), (2) 式可简化为

$$
\begin{gathered}
v_{/ /}=v_{0}\left(1+K_{/ /}\right), \\
v_{\perp}=v_{0}\left(1+K_{\perp}\right)+3 v_{Q}^{2} /\left(16 v_{0}\right),
\end{gathered}
$$


其中 $v_{Q}$ 是 $\eta=0$ 时的核四极共振 (NQR) 谱线频率, 或 NMR 中中心线与伴线的差频. $v_{0}$ 这里 定为 79.549MHz. 文献[6] 用 NQR 的方法给出 $v_{Q}(1)=19.75 \mathrm{MHz}$, 代人 (4) 式, 得出 $\mathrm{Cu}(1)$ 位 的 $K_{\mid}<0$. 例如 $295 \mathrm{~K}$ 时, $K_{i}=-0.437 \%$. 这显然是不合理的 ${ }^{[5}$. 我们采用文献[6] 中与我 们实验中有同样标称成分的掺铅 $\mathrm{Bi}$ 系 2223 相单相磁取向样品的 NMR 伴线测到的 $v_{Q}(1)=7.3$ $\mathrm{MHz}, v_{Q}(2)=19.4 \mathrm{MHz}$. 代人 (4) 式, 结合我们从实验得到的 $77 \mathrm{~K}$ 到 $295 \mathrm{~K}$ 之间谱线峰值的频 率位置数据, 可得到每个相应温度点的 $\mathrm{Cu}(1), \mathrm{Cu}(2)$ 位 $K_{/ /}, K_{\text {। }}$ 数据. 如图 2 所示. Knight 位移包括导电电子自旋贡献 $K^{S}$, 轨道贡献 $K^{L}$ 和在金属中很小的化学位移 $\sigma$ (可表示包含在 $K^{L}$ 之中), 即

$$
K_{a}(T)=K_{a}^{S}(T)+K_{a}^{L} \quad(\alpha=\perp, / /),
$$

其中 $K_{2}^{L}$ 是不随温度变化的. 所以 $K_{a}(T)$ 随温度变化实际上反映了 Knight 位移的 电子自旋贡献 $K_{a}^{S}(T)$ 随温度变化的规 律.

\section{3 核自旋 - 晶格弛豫率 $\left(1 / T_{1}\right)$ 的拟合}

$I=3 / 2$ 的 ${ }^{63} \mathrm{Cu}$ 中心线跃迁的核磁化 强度矢量经反转恢复趋于平衡态的变化 规律 ${ }^{[13]}$ :

$$
\begin{gathered}
M(t) / M(\infty)=1-0.2\left(\exp \left(-t / T_{1}\right)+\right. \\
\left.9 \exp \left(-6 t / T_{1}\right)\right),
\end{gathered}
$$

其中 $M(\infty)$ 表示平衡态的磁化强度矢 量. 在 $c$ 轴 $/ / B_{0}$ 时, $\mathrm{Cu}(1)$ 位和 $\mathrm{Cu}(2)$ 位 的谱线在整个温度变化范围内重叠. 考

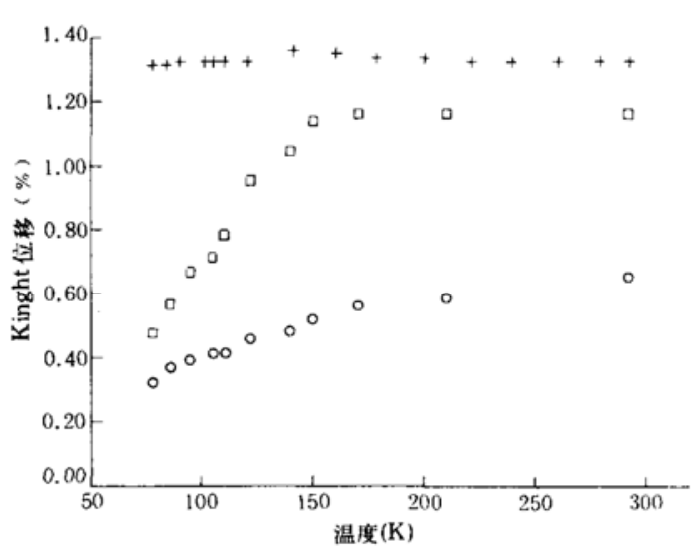

图 $2\left(\mathrm{Bi}_{1.6} \mathrm{~Pb}_{0.4}\right) \mathrm{Sr}_{2} \mathrm{Ca}_{2} \mathrm{Cu}_{3} \mathrm{O}_{\mathrm{y}}$ 磁取向样品的 ${ }^{63} \mathrm{CuKnight}$ 位移随温度变化关系

图中数据点: + 为晶粒 $c$ 轴平行于场时, $\mathrm{Cu}(1)$ 位和 $\mathrm{Cu}(2)$ 位 橧线; ○为晶粒 $c$ 轴垂直于磁场时, $\mathrm{Cu}(1)$ 位渞线; 口为晶粒 $c$ 轴垂直于磁场时, $\mathrm{Cu}(2)$ 位诸线

虑到 $c$ 轴 $\perp B_{0}$ 时, $\mathrm{Cu}(1)$ 和 $\mathrm{Cu}(2)$ 位的 $T_{1}$ 值之比约二倍左右, 有可能用二组分分解来拟合 $c$ 轴// $B_{0}$ 时的 $\mathrm{Cu}(1), \mathrm{Cu}(2)$ 位的 $T_{1}$ 值. 设 $\mathrm{Cu}(1), \mathrm{Cu}(2)$ 位磁化强度矢量的平衡值之比为 $M^{\prime}(\infty) /$ $M^{2}(\infty)=a .(6)$ 式的二组 分表达式为

$$
\begin{aligned}
M(t) / M_{0}= & 1-0.2 a\left(\exp \left(-t / T_{1}^{1}\right)+9 \exp \left(-6 t / T_{1}^{1}\right)\right) /(1+a) \\
& -0.2\left(\exp \left(-t / T_{1}^{2}\right)+9 \exp \left(-6 t / T_{1}^{2}\right)\right) /(1+a),
\end{aligned}
$$

其中 $M_{0}=M^{1}(\infty)+M^{2}(\infty) . a$ 是可调节参量. 通过反复逼近搜索, 总可找到最佳的 $a, T_{1}^{1}, T_{1}^{2}$ 值. 从晶体结构上考虑, $a$ 应该是 0.5 . 在 $140 \mathrm{~K}$ 时, 谱线中心位置左右半高宽范围内, 做多点 测量, 用已确定的 $T_{1}^{\mathrm{1}}, T_{1}^{2}$ 值 (谱线中心位置) 来拟合 $a$ 值. 发现 $a=0.57 \pm 0.11$, 说明 $\mathrm{Cu}(1)$ 和 $\mathrm{Cu}(2)$ 位的谱线中心是重叠的. 利用 (6), (7) 式, 得到了 $\mathrm{Cu}(1)$ 和 $\mathrm{Cu}(2)$ 位在晶粒 $c$ 轴平行 和垂直于磁场 2 种情况下的核自旋 - 晶格驰豫率, 如图 3 所示.

在 $c$ 轴 $\perp B_{0}$ 和 77.4, 100,200,292K 温度下, 测量 $\mathrm{Cu}(1)$ 和 $\mathrm{Cu}(2)$ 位的 ${ }^{65} \mathrm{Cu}$ 的 $T_{1}$ 值, 发现这二个位置的 $\left({ }^{65} T_{1} /{ }^{63} T_{1}\right)$ ，都在 $\left({ }^{63} \gamma /{ }^{65} \gamma\right)=0.87$ 附近, 而不是 $\left(e Q^{63} / e Q^{65}\right)=1.16$. 由此可说 明在77- $295 \mathrm{~K}$ 温度变化范围内, 核自旋是通过超精细磁相互作用与晶格中电子自旋发生能 量交换的. 

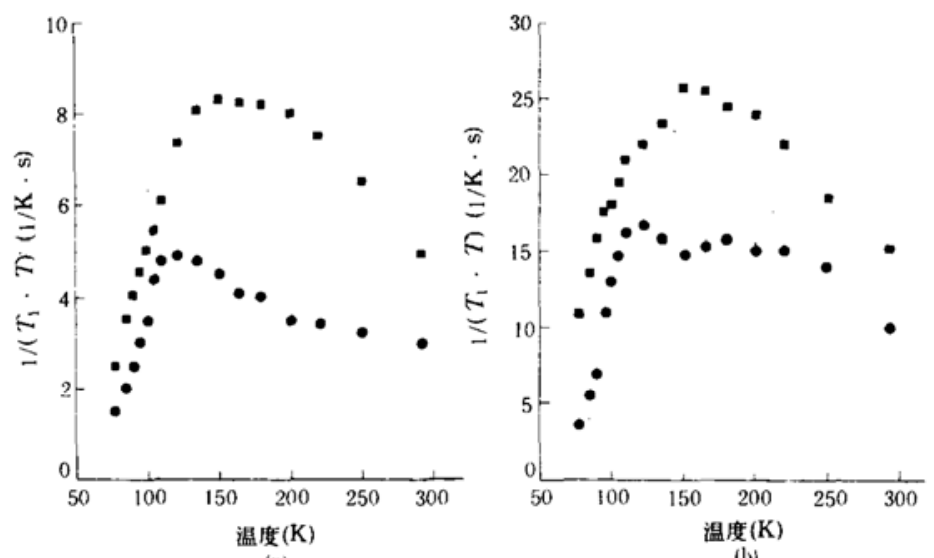

图 $3\left(\mathrm{Bi}_{1.6} \mathrm{~Pb}_{0.4}\right) \mathrm{Sr}_{2} \mathrm{Ca}_{2} \mathrm{Cu}_{3} \mathrm{O}_{\mathrm{y}}$ 磁取向样品的 ${ }^{63} \mathrm{Cu}$ 核自旋 - 晶格弛像率 $\left(1 / T_{1}\right)$ 随温度变化关系 图中数据点: - 为 $\mathrm{Cu}(1)$ 位谱线, - 为 $\mathrm{Cu}(2)$ 位橧线. (a) 晶粒 $c$ 轴平行于磁场时, (b) 晶粒 $c$ 轴垂直于磁场时

\section{3 结 果 讨 论}

从图 4 中发现 $\mathrm{Cu}(2)$ 位的 $K_{!}(T)$ 与 $\left(1 / T_{1} T\right)$ „在 $77-135 \mathrm{~K}$ 范围内随温度变化相

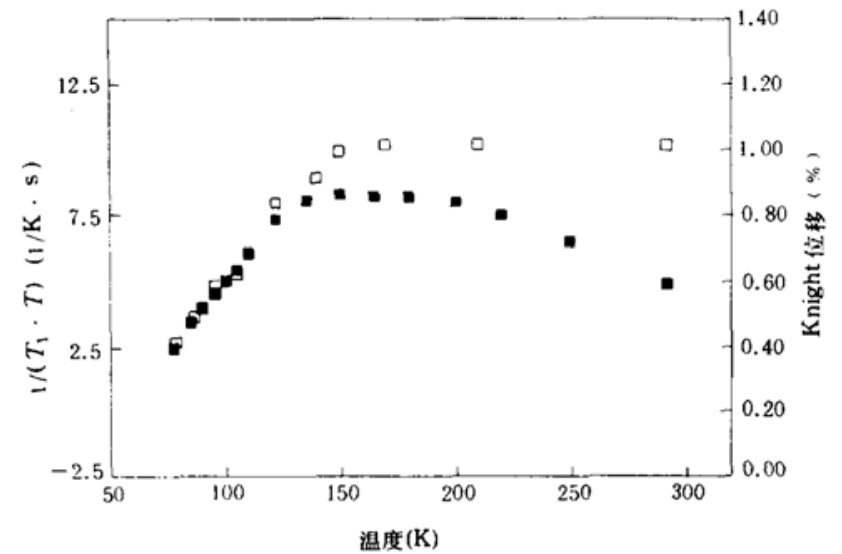

图 $4\left(\mathrm{Bi}_{1.6} \mathrm{~Pb}_{0.4}\right) \mathrm{Sr}_{2} \mathrm{Ca}_{2} \mathrm{Cu}_{3} \mathrm{O}_{\mathrm{y}}$ 取向样品中 $\mathrm{Cu}(2)$ 位的 $K_{\perp}(T)$ 与

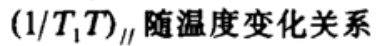

图中数据点: $\square$ 为 $K_{\perp}(T), \square$ 为 $\left(1 / T_{1} T\right)_{\|}$
同. 从图 2 和图 3 中也可发现 $\mathrm{Cu}(1)$ 位在 $77-110 \mathrm{~K}$ 范围内下 有类似现象. Walstedt 在 $Y$ 系 123 相缺氧样品 ${ }^{17}$ 和 $\mathrm{Bi}$ 系 2212 相样 品 ${ }^{[8]}$ 的 ${ }^{63} \mathrm{Cu}$ NMR 实验中发现, 在 100K 以下,

$$
\left(1 / T_{1} T\right)_{\|} \propto K_{\mid}{ }^{s}(T),
$$

文献 $[7,8]$ 对关系式 (8) 没作定量 的物理解释. 假设 $\mathrm{Bi}$ 系 2223 相 样品有这同样的规律 (其物理涵 义下一节再详细讨论), 我们用线 性回归计算 $\left(1 / T_{1} T\right)_{/ /}=a K_{\perp}^{S}(T)$ $=a K_{\perp}-a K_{\perp}^{L}$, 其中 $a$ 和 $K_{\perp}^{L}$ 都 是待求参数. 计算得到 $\mathrm{Cu}(1)$ 位 和 $\mathrm{Cu}(2)$ 位的电子轨道贡献 $K_{1}^{L}$

分别为 $0.25 \%$. 和 $0.20 \%$, 回归计算的线性相关系数为 0.93 和 0.99 . 从图 2 可知, $\mathrm{Cu}(1)$ 位和 $\mathrm{Cu}(2)$ 位的 $K_{/ /}(T)$ 在 $T_{\mathrm{c}}$ 上下到 $77 \mathrm{~K}$ 都无明显变化 (没做抗磁修正, 否则这种不依赖于 $T_{\mathrm{c}}$ 的效 应更加明显), 这样可以认为这些位的 Knight 位移自旋贡献 $K_{/ /}{ }^{s}$ 为零. 表 1 为 $295 \mathrm{~K}$ 时 $\mathrm{Cu}(1)$ 位和 $\mathrm{Cu}(2)$ 位 $\mathrm{Knight}$ 位移的电子轨道贡献和自旋贡献. $\mathrm{Cu}(1)$ 位和 $\mathrm{Cu}(2)$ 位轨道位移的垂直 分量与平行分量之比 $K_{\perp}{ }^{L} / K_{/ /}{ }^{L}$ 分别为 4.6 和 5.8. 这些数据非常接近 $Y$ 系 123 相中 $\mathrm{Cu}(2)$ 位 的 4.6 和 $\mathrm{Bi}$ 系 2212 相中 $\mathrm{Cu}$ 位的 $5.1^{[1.6)}$. 说明这些体系中 $\mathrm{Cu}^{++}$离子的晶体场参数是近似 的. 
表 $1295 \mathrm{~K}$ 时 Knight 位移的电子轨道贡献和自旋贡献

\begin{tabular}{clll}
\hline & & \multicolumn{1}{c}{$\mathrm{Cu}(1)$ 位 } & \multicolumn{1}{c}{$\mathrm{Cu}(2)$ 位 } \\
\hline 轨道贡㓓 & $K_{/ /}{ }^{2}(\%)$ & $1.160 \pm 0.013$ & $1.160 \pm 0.013$ \\
& $K_{\perp} Y(\%)$ & $0.250 \pm 0.025$ & $0.200 \pm 0.025$ \\
自旋贡献 & $K_{/ /}{ }^{s}(\%)$ & 0 & 0 \\
& $K_{\perp}{ }^{s}(\%)$ & $0.320 \pm 0.025$ & $0.820 \pm 0.025$ \\
\hline
\end{tabular}

在 $77-295 \mathrm{~K}$ 温度变化范围内, $\mathrm{Cu}$ (1)位和 $\mathrm{Cu}(2)$ 位的核自旋 - 晶格驰豫率 各向异性比值 $\left(1 / T_{1}\right)_{\perp} /\left(1 / T_{1}\right)_{/ /}$在 3.0-4.2 之间. 用能带理论计算各种超精细相互 作用引起的核自旋 - 晶格弛豫率, 其各向 异性之比总小于 $2.5^{[99}$. 在 $T_{c}$ 以上, $\mathrm{Cu}(1)$ 位 $\mathrm{Cu}(2)$ 位的核自旋 - 晶格驰豫率随温度变

化的关系不能用 Fermi 液体理论来解释, 即不满足 Korringa 关系:

$$
T_{1 / /} T\left(K_{\perp}^{S}(T)\right)^{2}=\text { 常数. }
$$

核自旋 - 晶格弛豫率在 $T_{c}$ 以上的增强可用 $\mathrm{Cu}$ 位格点电子自旋的反铁磁耦合和短程的自旋张 落关联来解释, 即所谓反铁磁 Fermi 液体唯象理论 (MMP 理论) ${ }^{1109}$. MMP 理论已很好地应用 于 $\mathrm{Y}$ 系和 $\mathrm{La}$ 系样品的核磁共振实验的定量分析 ${ }^{[11,12]}$. 根据我们对 $\mathrm{Bi}$ 系 2223 相样品 ${ }^{63} \mathrm{Cu},{ }^{17} \mathrm{O}$ $\mathrm{Knight}$ 位移的变温研究, $\mathrm{CuO}_{2}$ 平面的电子自旋磁化率是单分量的. MMP 理论给出:

$$
\left(1 / T_{1} T\right)=\frac{12 \pi}{\mu_{B}^{2} h^{2}} B^{2} k_{B}\left|\frac{x_{d}(T)}{\Gamma(T)}\left\|0.77+\left.\frac{\beta}{\pi^{2}}|1.83| \frac{\xi(T)}{a}\right|^{2}-\ln \left|\frac{\xi(T)}{a}\right|-0.3\right\|,\right.
$$

其中只有 $x_{0}(T), \xi(T), \Gamma(T)$ 是随度变化的唯象参数, $\Gamma(T)$ 是准粒子自旋胀落特征能, 在一定温 度范围内随温度变化很小, 可认为是常数 ${ }^{(11)} . \xi(T)$ 是反铁磁相关长度, 它的平均场形式为

$$
\xi^{2}(T)=\xi^{2}(0) T_{\mathrm{x}} /\left(T+T_{\mathrm{x}}\right),
$$

即在某一特征温度 $T_{\mathrm{x}}$ 以下随温度变化很小. $\chi_{\alpha}(T)$ 为电子自旋磁化率. 这样 (9)式在某一 $T_{\mathrm{x}}$ 温度下可近似地表示为

$$
\left(1 / T_{1} T\right) \propto \chi_{0}(T) .
$$

Knight 位移的自旋贡献 $K_{\alpha}^{s}(a=a, b, c)$ 可表示为 ${ }^{[s]}$

$$
K_{a}^{s}=A_{a} \chi d(T),
$$

其中 $A_{a}$ 为电子自旋与核的超精细场 $\alpha$ 分量, 它是不随温度变化的. 结合式 (9)一式 (12), 可以 得到在某一特征温度 $T_{\mathrm{X}}$ 下的关系式 (8). 所以 MMP 理论对 Walstedt 在 $\mathrm{Y}$ 系 123 相缺氧样 品, $\mathrm{Bi}$ 系 2212 相样品和我们在 $\mathrm{Bi}$ 系 2223 相样品中所发现的实验规律有很好的定量解释.

从图 2 和图 3 可看出, $\mathrm{Cu}(1)$ 位和 $\mathrm{Cu}(2)$ 位的特征温度和电子自旋磁化率 $\chi_{0}(T)$ 随温度 的变化关系的不同, 说明 $\mathrm{Cu}(1) \mathrm{O}_{2}$ 层和 $\mathrm{Cu}(2) \mathrm{O}_{2}$ 层的电子自旋动力学过程是不同的. ${ }^{17} \mathrm{O}$ 的 NMR 实验也说明了这一点.

\section{4 结 论}

正常 Fermi 液体物理图象的失效, 说明仅考虑长波长极限是不够的, 它是一种弱关联近 似. 而高 $T_{\mathrm{c}}$ 氧化物超导体是强关联体系. MMP 理论考虑了自旋的反铁磁峨合和短程的自旋 涨落关联, 定量地解释了体系的低频短波长磁学行为. 通过对 $\left(\mathrm{Bi}_{1.6} \mathrm{~Pb}_{0.4}\right) \mathrm{Sr}_{2} \mathrm{Ca}_{2} \mathrm{Cu}_{3} \mathrm{O}_{y}$ 磁取向 样品中 Knight 位移和核自旋- 晶格弛豫率的实验结果分析, 发现 (1) 在不同 $\mathrm{CuO}_{2}$ 层的特征温度 下, $\left(1 / T_{1} T\right)_{\|} \propto K_{\perp}{ }^{s}(T)$ 关系式成立. 这一结果说明解释正常态的 MMP 唯象理论能适用并延 
伸到临界温度以下的一定范围, 其物理原因还不清楚. (2) 分析得到 Knight 位移的电子轨道 贡献, 说明高 $T_{\mathrm{c}}$ 氧化物超导体体系中 $\mathrm{CuO}_{2}$ 平面上 $\mathrm{Cu}^{++}$离子的晶体场是近似的. (3) $\mathrm{Cu}(1)$ 位 和 $\mathrm{Cu}(2)$ 位的特征温度和电子自旋磁化率 $\chi_{0}(T)$ 随温度的变化关系的不同, 说明 $\mathrm{Cu}(1) \mathrm{O}_{2}$ 层和 $\mathrm{Cu}(2) \mathrm{O}_{2}$ 层的电子自旋动力学过程是不同的.

致谢样品制备是在中国科学院上海冶金研究所第三研究室进行的, 得到了傅耀先副研 究员、胡永祥工程师以及第三室其他老师的指教和帮助. 华东师范大学物理系俞永勤副教授 在 $T_{\mathrm{c}}$ 测量上给子了帮助.

\section{参考文献}

[1] Pennington, C. H., Slischter, C. P., Physical Properties of High Temperature Superconductors II (ed. Ginsherg, N. M. ), World Scientific, 1990, 269.

［2]董正超、歌学文等,华东师范大学学报 (自然科学版), 1991; (4): 33-41.

[3] Howes, A. P. et al., Physica. C, 1992, 193: 189.

[4] 许 亮、趴学文等,第七届全国波谱学学术会议论文摘要集 (2), 1992, 47.

[5] Carter, G. C. et al., Metallic Shifts in NMR, Pergamon, Oxford, 1979.

[6] Statt, B. W. et al., Physica, C, 1991, 183: 372.

[7] Walstedt, R. E. et al, Science, 1990, 248: 1082.

[ 8] Walstedt, R. E. et al., Phys. Rev., B, 1991, 44: 7760.

[ 9] Walstedt, R. E. et al., Phys. Rev., B, 1988, 38: 9299.

[10] Millis, A. J. et al., Phys. Rev., B, 1990, 42: 167.

[11] Monien, H. et al., Phys. Rev., B, 1991, 43: 258.

[12] Monien, H. et al., Phys. Rev., B, 1991, 43: 275.

[13] Narath, A., Phys. Rev., 1967, 162: 320. 